\title{
Cryptanalysis of the Stream Cipher DECIM^
}

\author{
Hongjun $\mathrm{Wu}$ and Bart Preneel \\ Katholieke Universiteit Leuven, ESAT/SCD-COSIC \\ Kasteelpark Arenberg 10, B-3001 Leuven-Heverlee, Belgium \\ \{wu.hongjun, bart.preneel\}@esat.kuleuven.be
}

\begin{abstract}
DECIM is a hardware oriented stream cipher with an 80-bit key and a 64-bit IV. In this paper, we point out two serious flaws in DECIM. One flaw is in the initialization of DECIM. It allows to recover about half of the key bits bit-by-bit when one key is used with about $2^{20}$ random IVs; only the first two bytes of each keystream are needed in the attack. The amount of computation required in the attack is negligible. Another flaw is in the keystream generation algorithm of DECIM. The keystream is heavily biased: any two adjacent keystream bits are equal with probability about $\frac{1}{2}+2^{-9}$. A message could be recovered from the ciphertext if that message is encrypted by DECIM for about $2^{18}$ times. DECIM with an 80 -bit key and an 80 -bit IV is also vulnerable to these attacks.
\end{abstract}

\section{Introduction}

DECIM [1] is a stream cipher that has been submitted to the ECRYPT stream cipher project [4]. The main feature of DECIM is the use of the ABSG decimation mechanism [1], an idea similar to the shrinking generator [36]. Another excellent feature is that a 32-bit buffer is used in DECIM to ensure that at each step DECIM generates one output bit.

In this paper, we point out two flaws in DECIM, one in the initialization algorithm, and another one in the keystream generation algorithm. The flaw in the initialization allows for any easy key recovery from the keystreams when one key is used with about $2^{20}$ random IVs. The flaw in the keystream generation algorithm results in a heavy bias in the keystream, hence the cipher is vulnerable to a broadcast attack.

In Sect. 2 we describe the DECIM cipher. Section 3 presents an key recovery attack on DECIM. The key recovery attack on DECIM is improved in Sect. 4. The broadcast attack on DECIM is described in Sect. 5. Section 6 shows that DECIM with an 80-bit IV is also vulnerable to the attacks. Section 7 concludes this paper.

\footnotetext{
* This work was supported in part by the Concerted Research Action (GOA) Ambiorics 2005/11 of the Flemish Government and in part by the European Commission through the IST Programme under Contract IST-2002-507932 ECRYPT.
} 


\section{Stream Cipher DECIM}

DECIM uses the ABSG decimation mechanism in the keystream generation in order to achieve high security and design simplicity. The keystream generation process and the key/IV setup are illustrated in Sect. 2.1 and 2.2, respectively.

\subsection{Keystream Generation}

The keystream generation diagram of DECIM is given in Fig. 1 DECIM has a regularly clocked LFSR which is defined by the feedback polynomial

$$
\begin{aligned}
P(X)= & X^{192}+X^{189}+X^{188}+X^{169}+X^{156}+X^{155}+X^{132}+ \\
& X^{131}+X^{94}+X^{77}+X^{46}+X^{17}+X^{16}+X^{5}+1
\end{aligned}
$$

over $G F(2)$. The related recursion is given as

$$
\begin{aligned}
& s_{192+n}= s_{187+n} \oplus s_{176+n} \oplus s_{175+n} \oplus s_{146+n} \oplus s_{115+n} \oplus s_{98+n} \oplus s_{61+n} \\
& \oplus s_{60+n} \oplus s_{37+n} \oplus s_{36+n} \oplus s_{23+n} \oplus s_{4+n} \oplus s_{3+n} \oplus s_{n} .
\end{aligned}
$$

At each stage, two bits are generated from the LFSR as follows:

$$
\begin{gathered}
y_{t, 1}=f\left(s_{t+1}, s_{t+32}, s_{t+40}, s_{t+101}, s_{t+164}, s_{t+178}, s_{t+187}\right), \\
y_{t, 2}=f\left(s_{t+6}, s_{t+8}, s_{t+60}, s_{t+116}, s_{t+145}, s_{t+181}, s_{t+191}\right),
\end{gathered}
$$

where the Boolean function $f$ is defined as

$$
f\left(x_{i_{1}}, \ldots, x_{i_{7}}\right)=\sum_{1 \leq j<k \leq 7} x_{i_{j}} x_{i_{k}} .
$$

The binary sequence $y$ consists of all the $y_{t, 1}$ and $y_{t, 2}$ as

$$
y=y_{0,1} y_{0,2} y_{1,1} y_{1,2} \cdots y_{t, 1} y_{t, 2} \cdots
$$

The keystream sequence $z$ is generated from the binary sequence $y$ through the ABSG decimation algorithm. The sequence $y$ is split into subsequences of the form $\left(\bar{b}, b^{i}, \bar{b}\right)$, with $i \geq 0$ and $b \in\{0,1\} ; \bar{b}$ denotes the complement of $b$ in $\{0,1\}$. For every subsequence $\left(\bar{b}, b^{i}, \bar{b}\right)$, the output bit is $b$ for $i=0$, and $\bar{b}$ otherwise. The ABSG algorithm is given below

$$
\begin{aligned}
& \text { Input: }\left(y_{0}, y_{1}, \ldots\right) \\
& \text { Set: } i \leftarrow 0 ; j \leftarrow 0 ; \\
& \text { Repeat the following steps: } \\
& \qquad \begin{array}{r}
\quad \leftarrow y_{i}, z_{j} \leftarrow y_{i+1}, i \leftarrow i+1 ; \\
\quad \text { while }\left(y_{i}=\bar{e}\right) i \leftarrow i+1 ; \\
\quad i \leftarrow i+1 ; \text { output } z_{j} ; j \leftarrow j+1 ;
\end{array}
\end{aligned}
$$

Remarks. The above description of the ABSG and the pseudo-code of ABSG are quoted from [1]. However the outputs of the pseudo-code are the complements 


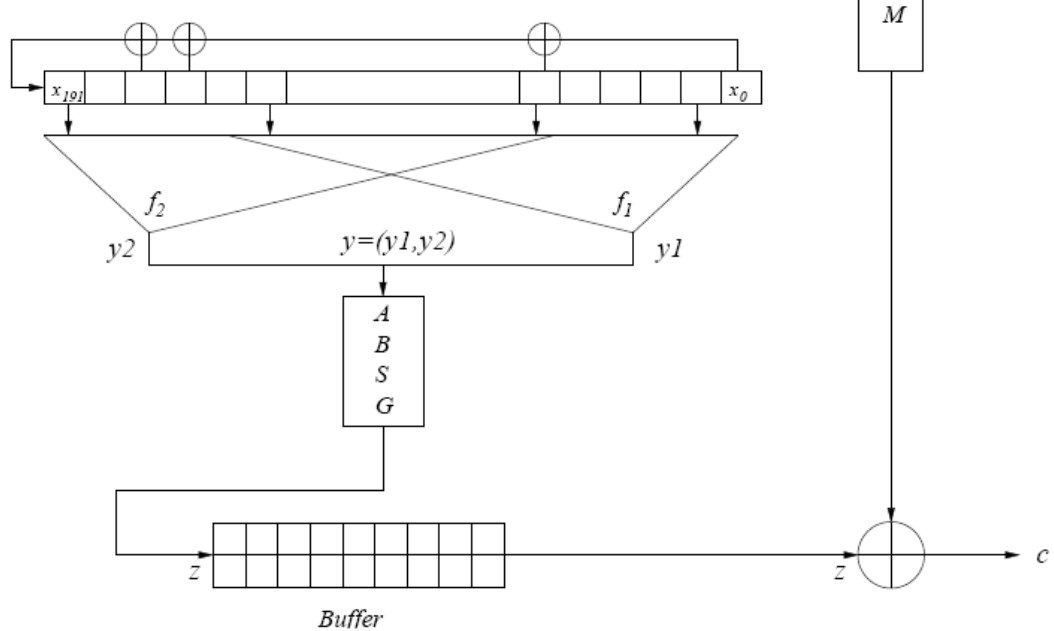

Fig. 1. Keystream Generation Diagram of DECIM [1]

of that of the ABSG algorithm. Anyway, this difference has no effect on the security of DECIM. In the rest of the paper, we assume that the DECIM uses the pseudo-code of ABSG given above.

DECIM is designed to output one bit every two stages. A 32-bit buffer is used to ensure that the probability that there is no output bit is extremely small $\left(2^{-89}\right)$.

\subsection{Initialization}

The secret key $K$ is an 80-bit key. The 64-bit IV is expanded to an 80-bit vector by adding zeros from position 64 up to position 79 . The initial value of the LFSR state is loaded as follows

$$
s_{i}= \begin{cases}K_{i} \vee I V_{i} & \text { for } 0 \leq i \leq 55 \\ K_{i-56} \wedge \overline{I V_{i-56}} & \text { for } 56 \leq i \leq 111 \\ K_{i-112} \oplus I V_{i-112} & \text { for } 112 \leq i \leq 191\end{cases}
$$

The LFSR is clocked 192 times. After the $t$-th clocking, $y_{t, 1}$ and $y_{t, 2}$ are XORed to the $x_{t, 192}$ as

$$
s_{t+192}=s_{t+192} \oplus y_{t, 1} \oplus y_{t, 2} .
$$

Then one of two permutations $\pi_{1}$ and $\pi_{2}$ is applied to permute 7 elements $s_{t+5}$, $s_{t+31}, s_{t+59}, s_{t+100}, s_{t+144}, s_{t+177}, s_{t+186}$. Two bits $y_{t, 1}$ and $y_{t, 2}$ are input to the ABSG, if the output of the ABSG is 1 , then $\pi_{1}$ is applied; if the output of the ABSG is 0 or if there is no output, then $\pi_{2}$ is applied. The two permutations are defined as

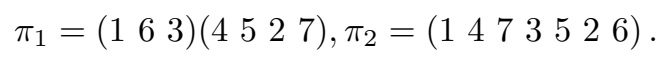




\section{Key Recovery Attack on DECIM}

In this section, we develop attacks to recover the secret key of DECIM. This non-optimized attack applies when the same secret key is used with a number of random IVs, and the first 3 bytes of each keystream are known. The optimized attack is given in the next section.

\subsection{The Effects of the Permutations $\pi_{1}$ and $\pi_{2}$}

The two permutations in the initialization stage of DECIM provide high nonlinearity to the initialization process. However, the permutations also cause some bits in the LFSR to be updated in an improper way. This has a very negative impact on the security of DECIM.

The permutation $\pi_{1}$ is poorly designed. In order to investigate the effects of this permutation, we analyze a weak version by assuming that only this permutation is used in the initialization process, i.e., we replace $\pi_{2}$ with $\pi_{1}$. The values of 140 elements in the $\operatorname{LFSR}\left(s_{5}, s_{6}, \ldots, s_{58}\right.$, and $\left.s_{100}, s_{101}, \ldots, s_{185}\right)$ would never be updated by the initialization process. For example, $s_{21}$ would always become $s_{192+6}$. The details are given below. We trace the bit $s_{21}$, after 16 steps it becomes $s_{16+5}$ due to the shift of the LFSR. Then it becomes $s_{16+177}$ due to the permutation $\pi_{1}$. After 33 steps, it becomes $s_{49+144}$ due to the shift of the LFSR. Then it becomes $s_{49+31}$ due to the permutation $\pi_{1}$. After 26 steps, it becomes $s_{75+5}$ due to the shift of the LFSR. Then it becomes $s_{75+177}$ due to the permutation $\pi_{1}$. This process repeats and at the end of the initialization process, it becomes $s_{192+6}$.

The first bit of the keystream is given as $y_{192,2}$; it is computed as $y_{192,2}=$ $f\left(s_{192+6}, s_{192+8}, s_{192+60}, s_{192+116}, s_{192+145}, s_{192+181}, s_{192+191}\right)$. By tracing the bits of the LFSR during the initialization process, we know that $s_{192+6} \Leftarrow s_{21}$, $s_{192+8} \Leftarrow s_{23}, s_{192+116} \Leftarrow s_{132}, s_{192+145} \Leftarrow s_{160}, s_{192+181} \Leftarrow s_{33}$. If every key and IV pair is randomly generated, then according to the loading of the key and IV, we know that $s_{21}, s_{23}$, and $s_{33}$ take value 1 with probability 0.75 . Thus according to the definition of the function $f$, the value of $y_{192,2}$ is 0 with probability 0.582 . So the first bit of the keystream is heavily biased. It shows that the effect of the permutation $\pi_{1}$ is terrible.

In DECIM, there are two permutations, $\pi_{1}$ and $\pi_{2}$. They are chosen according to the output of ABSG: $\pi_{1}$ is chosen with probability $\frac{1}{3}, \pi_{2}$ with probability $\frac{2}{3}$. Due to these two permutations, the number of bits that are not updated by the initialization process is reduced to 54.5 (obtained by running $2^{16}$ random key and IV pairs). It shows that the permutations $\pi_{1}$ and $\pi_{2}$ which are chosen by the output of ABSG have a negative impact on the security of DECIM.

\section{$3.2 \quad$ Recovering $K_{21}$}

In the initialization process, we monitor the bit $s_{21} \cdot s_{21}$ becomes $s_{192+6}$ with probability $\frac{1}{27}$. If $s_{192+6}$ takes the value 0 , and all the other bits in the LFSR at the 192-th step are distributed uniformly, then the value of the first bit of the 
keystream is 0 with probability $q_{0}=\frac{56}{128}$. If $s_{192+6}$ is takes the value 1 , and all the other bits of the LFSR at the 192-th step are distributed uniformly, then the value of the first bit of the keystream is 0 with probability $q_{1}=\frac{72}{128}$. Denote the probability that the value of the first keystream bit is 0 when $s_{21}=0$ as $p_{0}$, and the probability that the value of the first keystream bit is 0 when $s_{21}=1$ as $p_{1}$. Then $\Delta p=p_{1}-p_{0}=\frac{1}{27} \times\left(q_{1}-q_{0}\right)=2^{-7.75}$. In an experiment we chose $2^{20}$ random IVs for $s_{21}=0$, and another $2^{20}$ random IVs for $s_{21}=1$, and we found that $\Delta p=2^{-7.99}$. The experimental result confirms that the theoretical result $\Delta p=2^{-7.75}$ is correct.

The above property can be applied to recover $K_{21}$ as follows. Suppose that the same key is used with $N$ random IVs to generate keystreams. For the keystreams with $I V_{21}=0$, we compute the probability that the value of the first bit is 0 , and denote this probability as $p_{0}^{\prime}$. For the keystreams with $I V_{21}=1$, we compute the probability that the value of the first bit is 0 , and denote this probability as $p_{1}^{\prime}$. If $p_{1}^{\prime}>p_{0}^{\prime}$, we decide that $K_{21}=0$; otherwise, $K_{21}=1$. For $N=\left(\frac{\Delta p}{2}\right)^{-2} \times 2=2^{18.5}$, the attack can determine the value of $K_{21}$ with success rate 0.977 .

\section{$3.3 \quad$ Recovering $K_{22} K_{23} \ldots K_{30}$}

By tracing the bits in the initialization process, we notice that each $s_{22+i}$ is mapped to $s_{192+7+i}$ with probability $\frac{1}{27}$ for $0 \leq i \leq 8$ (each of them is only mapped by $\pi_{1}$ at $\left.s_{t+5}\right)$. We know that $s_{22+i}=K_{22+i} \vee I V_{22+i}$, and $s_{192+7+i}$, $s_{192+9+i}$ are used in the generation of $y_{193+i, 2}$ for $0 \leq i \leq 10$. In this section, we show that the key bits $K_{22} K_{23} K_{24} \ldots K_{30}$ can be recovered from the keystream.

An attack similar to that given in Sect. 3.2 can be applied to recover the value of $K_{23}$ from the first keystream bits generated from $2^{18.5}$ IVs.

In order to determine the values of $K_{22}$ and $K_{24}$, we observe the second bit of the keystream. Due to the disturbance of the ABSG, $y_{193,2}$ becomes the second keystream bit with probability 0.5 . Thus $\Delta p^{\prime}=0.5 \times \Delta p=2^{-8.75}$. To recover $K_{22}$ and $K_{24}$, we need $2^{20.5}$ IVs in order to obtain a success probability of 0.977 .

In order to determine the value of $K_{25}$, we observe the second and third bits of the keystream. $y_{194,2}$ becomes the second bit of the keystream with probability $\frac{1}{8}$, and becomes the third bit of the keystream with probability $\frac{1}{4}$. Thus $\Delta p^{\prime \prime}=$ $\frac{1}{2} \times\left(\frac{1}{4}+\frac{1}{8}\right) \times \Delta p=2^{-10.165}$. To recover $K_{25}$, we need $2^{22.3}$ IVs in order to obtain a success probability of 0.977 .

We omit the details of recovering $K_{26} \cdots K_{29}$. To recover $K_{30}$, we observe the fifth, sixth and seventh bits of the keystream. $y_{199,2}$ would become one of these three bits with probability $\frac{77}{256}$. Thus $\Delta p^{\prime \prime \prime}=\frac{1}{3} \times \frac{77}{256} \times \Delta p=2^{-11.068}$. To recover $K_{29}$, we need $2^{23.5514} \mathrm{IVs}$ in order to obtain the success rate 0.977 .

\subsection{Recovering $K_{9} K_{10} \ldots K_{19}$}

By tracing the bits in the initialization process, we notice that each $s_{9+i}$ is mapped to $s_{192+166+i}$ with probability $\frac{1}{27}$ for $0 \leq i \leq 10$ (each of them is only mapped by $\pi_{1}$ at $\left.s_{t+5}\right)$. We know that $s_{9+i}=K_{9+i} \vee I V_{9+i}$, and $s_{192+166+i}$ is 
used in the generation of $y_{194+i, 1}$ for $0 \leq i \leq 10$. The attacks given in this section are similar to those given above. We only illustrate how to recover $K_{9}$ and $K_{19}$.

In order to determine the value of $K_{9}$, we observe the second bit of the keystream. $y_{194,1}$ becomes the second bit of the keystream with probability $\frac{1}{4}$. Thus $\Delta p^{(4)}=\frac{1}{4} \times \Delta p=2^{-9.75}$. To recover $K_{9}$, we need $2^{22.5}$ IVs in order to obtain a success probability of 0.977 .

In order to determine the value of $K_{19}$, we observe the 8-th, 9-th and 10-th bits of the keystream. $y_{204,1}$ becomes one of these three bits with probability 0.25966 . Thus $\Delta p^{(5)}=\frac{1}{3} \times 0.25966 \times \Delta p=2^{-11.28}$. To recover $K_{19}$, we need $2^{23.98} \mathrm{IVs}$ in order to obtain a success probability of 0.977 .

\subsection{Recovering $K_{32} K_{33} \ldots K_{46}$}

By tracing the bits in the initialization process, we notice that each $s_{144+i}$ is mapped to $s_{192+16+i}$ with probability $\frac{1}{27}$ for $0 \leq i \leq 14$ (each of them is only mapped by $\pi_{1}$ at $\left.s_{t+5}\right)$. We know that $s_{144+i}=K_{32+i} \oplus I V_{32+i}$, and $s_{192+16+i}$ is used in the generation of $y_{200+i, 1}$ for $0 \leq i \leq 14$.

Since for $s_{144+i}(0 \leq i \leq 14)$, the key bits are XORed with the IV bits, the attack is slightly modified. For example, if the probability of 0 in the keystream for $I V_{32}=0$ is higher than the probability of 0 in the keystream for $I V_{32}=1$, then we predict that $K_{32}=0$; otherwise, $K_{32}=1$. We only illustrate how to recover $K_{32}$ and $K_{46}$.

In order to determine the value of $K_{32}$, we observe the sixth, seventh and eighth bits of the keystream. $y_{200,2}$ becomes one of these three bits with probability 0.28027 . Thus $\Delta p^{(6)}=\frac{1}{3} \times 0.28027 \times \Delta p=2^{-11.17}$. To recover $K_{32}$, we need $2^{23.755}$ IVs in order to obtain a success probability of 0.977 .

In order to determine the value of $K_{46}$, we assume that starting from the fourth bit of the sequence $y$, each bit becomes the output with probability $\frac{1}{3}$. Then $y_{214,2}$ becomes one of the 12th, 13th, .., 18th bits of the keystream with probability 0.16637 . Thus $\Delta p^{(7)}=\frac{1}{7} \times 0.16637 \times \Delta p=2^{-13.145}$. To recover $K_{29}$, we need $2^{26.482} \mathrm{IVs}$ in order to obtain a success probability of 0.977 .

The attacks given in this section recover 36 bits of the secret key with about $2^{26}$ random IVs. For each IV, only the first 3 bytes of the keystream are needed in the attack.

\section{Improving the Key Recovery Attack}

In the above attacks, we deal with the bits affected only by $\pi_{1}$ at $s_{t+5}$ during the initialization (the bits affected by $\pi_{2}$ are not considered in the attack). In order to improve the attack, we have used a computer program to trace all the possibilities for each bit $s_{i}(0 \leq i \leq 175)$ during the initialization process to find out the distribution of that bit at the end of initialization. Then we have searched the optimal attack for that bit. We have performed the experiment, and found that 44 key bits can be recovered with less than $2^{20}$ IVs, and only the first 2 bytes of the keystream are required in the attack. The experiment results are given in Table 1 in Appendix A. 


\section{The Keystream of DECIM Is Heavily Biased}

The nonlinear function $f$ in DECIM is extremely simple. However this Boolean function is balanced but not 1-resilent. Unfortunately the ABSG decimation mechanism and the buffer in the output function fail to eliminate the bias existing in the output of $f$, hence the keystream is heavily biased.

\subsection{The Keystream Is Biased}

We start with analyzing the function $f$

$$
f\left(x_{i_{1}}, \ldots, x_{i_{7}}\right)=\sum_{1 \leq j<k \leq 7} x_{i_{j}} x_{i_{k}} .
$$

If any bit of the input of $f$ is equal to 1 , then $f$ outputs a ' 1 ' with probability $\frac{72}{128}$; otherwise it outputs a ' 1 ' with probability $\frac{56}{128}$. Thus for $f\left(x_{i_{1}}, \ldots, x_{i_{7}}\right)$ and $f\left(x_{i_{1}}^{\prime}, \ldots, x_{i_{7}}^{\prime}\right)$, if one bit of one input is always equal to one bit of another input (i.e., $x_{i_{a}}=x_{i_{b}}^{\prime}$ where $0 \leq a, b \leq 7$ ), then the outputs related to these two inputs would be equal with probability $\left(\frac{56}{128}\right)^{2}+\left(\frac{72}{128}\right)^{2}=\frac{65}{128}$.

Note that $y_{t, 1}$ and $y_{t, 2}$ are computed as follows

$$
\begin{gathered}
y_{t, 1}=f\left(s_{t+1}, s_{t+32}, s_{t+40}, s_{t+101}, s_{t+164}, s_{t+178}, s_{t+187}\right), \\
y_{t, 2}=f\left(s_{t+6}, s_{t+8}, s_{t+60}, s_{t+116}, s_{t+145}, s_{t+181}, s_{t+191}\right) .
\end{gathered}
$$

Denote $A=\{1,32,40,101,164,178,187\}, B=\{6,8,60,116,145,181,191\}$, and denote each element of $A$ by $a_{i}$, and each element of $B$ by $b_{i}(1 \leq i \leq 7)$. Then $y_{t, 1}=y_{t+a_{i}-a_{j}, 1}$ and $y_{t, 2}=y_{t+b_{i}-b_{j}, 2}$ with probability $\frac{65}{128}$ for $1 \leq i, j \leq 7$ and $i \neq j$. And $y_{t+b_{i}-a_{j}, 1}=y_{t, 2}$ with probability $\frac{65}{128}$ for $1 \leq i, j \leq 7$. It shows that the binary sequence $y$ is heavily biased.

The heavily biased sequence $y$ is used as input to the ABSG decimation algorithm. It results in a heavily biased output. In the attack, we are interested in those biases in $y$ that would not be significantly reduced by the ABSG Algorithm. Thus we will analyze the bias of $\left(y_{t+3,1}, y_{t, 2}\right),\left(y_{t+4,1}, y_{t, 2}\right)$ and $\left(y_{t, 2}, y_{t+2,2}\right)$ to find out how they affect the randomness of the output of ABSG.

For example, we analyze the effect of the bias of $\left(y_{t+3,1}, y_{t, 2}\right) \cdot y_{t+3,1}=y_{t, 2}$ with probability $\frac{65}{128}$. Denote the $i$-th bit of the sequence $y$ by $y^{i}$. Thus $y^{i}=y^{i+5}$ with probability $\frac{129}{256} \cdot\left(y^{i}, y^{i+5}\right)$ would affect the bias of the output of the ABSG in two approaches. One approach is that $\left(y^{i}, y^{i+5}\right)$ becomes $\left(z_{j}, z_{j+2}\right)$ with probability $\frac{1}{4}$ (case 1: $y_{i}=y_{i-1}, y_{i+2} \neq y_{i+1}$ and $y_{i+3}=y_{i+2}$; case $2: y_{i} \neq y_{i-1}, y_{i+1}=y_{i-1}$ and $\left.y_{i+3}=y_{i+2}\right)$. Thus for this approach, the bias of $\left(y^{i}, y^{i+5}\right)$ causes $z_{j}=z_{j+2}$ with probability $\frac{513}{1024}$. Another approach is that if $y_{i}=y_{i-1}$ and $y_{i+2}=y_{i+1}$, then $\left(y_{i}, y_{i+4}\right)$ becomes $\left(z_{j}, z_{j+2}\right)$. Note that $y_{i+4}=y_{i-1}$ with probability $\frac{129}{256}$, so $z_{j}=z_{j+2}$ with probability $\frac{129}{256}$. This approach happens with probability $\frac{1}{4}$. Thus the bias of $\left(y^{i}, y^{i+5}\right)$ causes $z_{j}=z_{j+2}$ with probability $\frac{513}{1024}$. Combining these two approaches, we know that $z_{j}=z_{j+2}$ with probability $\frac{257}{512}$.

We continue analyzing the above example since the output of ABSG decimation algorithm should pass through the buffer before becoming keystream. 
By analyzing the ABSG decimation algorithm and the buffer, we notice that if $\left(y^{i}, y^{i+5}\right)$ becomes $z_{j}=z_{j+2}$ after the ABSG decimation algorithm, then it becomes $z_{k}^{\prime}=z_{k+1}^{\prime}$ with probability 0.6135 after passing through the buffer; if $\left(y^{i}, y^{i+4}\right)$ becomes $z_{j}=z_{j+2}$ after the ABSG decimation algorithm, then it becomes $z_{k}^{\prime}=z_{k+1}^{\prime}$ with probability 0.5189 after passing through the buffer. Thus after passing through the buffer, the two approaches lead to $z_{k}^{\prime}=z_{k+1}^{\prime}$ with probability $\frac{1}{2}+0.6135 \times \frac{1}{1024}+0.5189 \times \frac{1}{1024}=\frac{1}{2}+2^{-9.82}$.

A similar analysis can be applied to the biases resulting from $\left(y_{t+4,1}, y_{t, 2}\right)$ and $\left(y_{t, 2}, y_{t+2,2}\right)$. The bias of $\left(y_{t, 2}, y_{t+2,2}\right)$ would cause $z_{k}^{\prime}=z_{k+1}^{\prime}$ with probability about $\frac{1}{2}+2^{-10.84}$, and the bias of $\left(y_{t+4,1}, y_{t, 2}\right)$ would cause $z_{k}^{\prime}=z_{k+1}^{\prime}$ with probability about $\frac{1}{2}+2^{-11.73}$.

Combining the effects of $\left(y_{t+3,1}, y_{t, 2}\right),\left(y_{t+4,1}, y_{t, 2}\right)$ and $\left(y_{t, 2}, y_{t+2,2}\right)$, the bias of $z_{k}^{\prime}=z_{k+1}^{\prime}$ is about $\frac{1}{2}+2^{-9.82}+2^{-10.84}+2^{-11.73}=\frac{1}{2}+2^{-9.00}$.

Now we verify the above analysis with an experiment. We have generated about $2^{30}$ keystream bits from DECIM and found that $z_{k}^{\prime}=z_{k+1}^{\prime}$ is about $\frac{1}{2}+2^{-8.67}$. The experimental result shows that the theoretical result is close to that obtained from the experiment.

\subsection{Broadcast Attack}

Due to the bias in the keystream, part of the message could be recovered from the ciphertexts if the same message is encrypted many times using DECIM with random key and IV pairs. A similar attack has been applied to RC4 by Mantin and Shamir [5].

Suppose that one message bit is encrypted $N$ times, and each keystream bit is 0 with probability $\frac{1}{2}+\Delta p$ with $\Delta p>0$. Denote the number of ' 0 ' in the ciphertext bits by $n_{0}$. If $n_{0}>\frac{N}{2}$, we conclude that the message bit is equal to ' 0 '; otherwise, we conclude that the message bit is equal to ' 1 '. For $N=\Delta p^{-2}$, the message bit is recovered with a success probability of 0.977 .

Thus if one message is encrypted about $2^{18}$ times with different keys and IVs, the message could be recovered from the ciphertexts.

\section{Attacks on DECIM with 80-bit IV}

The keystream generation algorithm of DECIM with an 80-bit IV is the same as DECIM with a 64-bit IV. Thus DECIM with an 80-bit IV still generates heavily biased keystream and it is vulnerable to the broadcast attack.

The initialization process of DECIM with an 80-bit IV is slightly different from the 64-bit IV version. The key and IV are loaded into the LFSR as

$$
s_{i}= \begin{cases}0 & \text { for } 0 \leq i \leq 31 \\ K_{i-32} \oplus I V_{i-32} & \text { for } 32 \leq i \leq 111 \\ K_{i-112} & \text { for } 112 \leq i \leq 191\end{cases}
$$

Similar to the attack given in Sect. 4, we have carried out an experiment to compute the IVs required to recover each bit. With $2^{21} \mathrm{IVs}, 41$ bits of the secret 
key could be recovered. Only the first 2 bytes of the keystream are required in the attack. The experiment results are given in Table 2 in Appendix A.

\section{Conclusion}

In this paper, we have developed two attacks against the stream cipher DECIM. The key could be recovered easily from the keystream with about $2^{20}$ random IVs. And the keystream of DECIM is heavily biased. The results indicate that DECIM is very weak.

Recently, the designers of DECIM have proposed DECIM v2 [2]. DECIM v2 is much simpler than DECIM. The initialization of DECIM v2 uses 768 steps of the keystream generation algorithm with the output bit being XORed to the LFSR. The filter is changed and $f$ is one-resilient. DECIM v2 is not vulnerable to the attacks presented in this paper.

\section{Acknowledgements}

The authors would like to thank the anonymous reviewers for their helpful comments.

\section{References}

1. C. Berbain, O. Billet, A. Canteaut, N. Courtois, B. Debraize, H. Gilbert, L. Goubin, A. Gouget, L. Granboulan, C. Lauradoux, M. Minier, T. Pornin, H. Sibert. "Decim A New Stream Cipher for Hardware Applications," ECRYPT Stream Cipher Project Report 2005/004. Available at http://www.ecrypt.eu.org/stream/

2. C. Berbain, O. Billet, A. Canteaut, N. Courtois, B. Debraize, H. Gilbert, L. Goubin, A. Gouget, L. Granboulan, C. Lauradoux, M. Minier, T. Pornin, H. Sibert. "DECIM v2," ECRYPT Stream Cipher Project Report 2006/004. Available at http://www.ecrypt.eu.org/stream/

3. D. Coppersmith, H. Krawczyk, and Y. Mansour. "The Shrinking Generator," in Advances in Cryptology - CRYPTO'93, volume 773 of Lecture Notes in Computer Science, pages 22-39. Springer-Verlag, 1993.

4. ECRYPT Stream Cipher Project, at http://www.ecrypt.eu.org/stream/

5. I. Mantin, A. Shamir. "A Practical Attack on Broadcast RC4," in Fast Software Encryption (FSE 2001), LNCS2335, pp. 152-164, Springer-Verlag, 2001.

6. W. Meier and O. Staffelbach. "The Self-Shrinking Generator," in Advances in Cryptology - EUROCRYPT'94, volume 950 of Lecture Notes in Computer Science, pages 205-214, Springer-Verlag, 1994.

\section{A The Number of IVs Required to Break DECIM}

Table 1 gives the number of IVs required to break DECIM with a 64-bit IV. 44 key bits can be recovered with less than $2^{20}$ IVs. Table 2 gives the number of 
Table 1. Number of IVs required to recover the key bits (64-bit IV)

\begin{tabular}{|c|c|c|c|c|c|}
\hline & Affected Bits & $\begin{array}{l}\text { Amount of } \\
\text { IVs }\left(\log _{2}\right)\end{array}$ & & Affected Bits & $\begin{array}{l}\text { Amount of } \\
\text { IVs }\left(\log _{2}\right)\end{array}$ \\
\hline$K_{0}$ & $s_{112} \Rightarrow s_{192+60}$ & 18.95 & $\overline{K_{1}}$ & $s_{57} \Rightarrow s_{192+122}$ & 20.83 \\
\hline$K_{2}$ & $s_{58} \Rightarrow s_{192+116}$ & 18.80 & $K_{3}$ & $s_{115} \Rightarrow s_{192+104}$ & 20.46 \\
\hline$K_{4}$ & $s_{116} \Rightarrow s_{192+105}$ & 21.41 & $K_{5}$ & $s_{117} \Rightarrow s_{192+106}$ & 21.54 \\
\hline$K_{6}$ & $s_{118} \Rightarrow s_{192+107}$ & 21.67 & $K_{7}$ & $s_{119} \Rightarrow s_{192+108}$ & 21.72 \\
\hline$K_{8}$ & $s_{120} \Rightarrow s_{192+145}$ & 21.21 & $K_{9}$ & $s_{121} \Rightarrow s_{192+110}$ & 21.92 \\
\hline$K_{10}$ & $s_{10} \Rightarrow s_{192+116}$ & 17.69 & $K_{11}$ & $s_{11} \Rightarrow s_{192+117}$ & 19.62 \\
\hline$K_{12}$ & $s_{68} \Rightarrow s_{192+6}$ & 18.88 & $K_{13}$ & $s_{69} \Rightarrow s_{192+7}$ & 20.82 \\
\hline$K_{14}$ & $s_{70} \Rightarrow s_{192+8}$ & 18.82 & $K_{15}$ & $s_{127} \Rightarrow s_{192+116}$ & 16.66 \\
\hline$K_{16}$ & $s_{128} \Rightarrow s_{192+117}$ & 18.70 & $K_{17}$ & $s_{17} \Rightarrow s_{192+6}$ & 16.92 \\
\hline $\bar{K} K_{18}$ & $s_{18} \Rightarrow s_{192+7}$ & 18.82 & $K_{19}$ & $s_{19} \Rightarrow s_{192+8}$ & 16.80 \\
\hline$\overline{K_{20}}$ & $s_{20} \Rightarrow s_{192+9}$ & 18.73 & $\overline{K_{21}}$ & $s_{21} \Rightarrow s_{192+6}$ & 18.59 \\
\hline$K_{22}$ & $s_{22} \Rightarrow s_{192+7}$ & 20.67 & $K_{23}$ & $s_{23} \Rightarrow s_{192+8}$ & 18.70 \\
\hline$\overline{K_{24}}$ & $s_{80} \Rightarrow s_{192+146}$ & 20.80 & $\overline{K_{25}}$ & $s_{25} \Rightarrow s_{192+116}$ & 17.97 \\
\hline$\overline{K_{26}}$ & $s_{138} \Rightarrow s_{192+6}$ & 17.79 & $\overline{K_{27}}$ & $s_{139} \Rightarrow s_{192+7}$ & 19.87 \\
\hline$K_{28}$ & $s_{140} \Rightarrow s_{192+8}$ & 17.86 & $K_{29}$ & $s_{141} \Rightarrow s_{192+9}$ & 19.67 \\
\hline$K_{30}$ & $s_{142} \Rightarrow s_{192+10}$ & 21.46 & $K_{31}$ & $s_{31} \Rightarrow s_{192+182}$ & 18.36 \\
\hline$K_{32}$ & $s_{32} \Rightarrow s_{192+183}$ & 20.70 & $K_{33}$ & $s_{33} \Rightarrow s_{192+113}$ & 20.97 \\
\hline$K_{34}$ & $s_{34} \Rightarrow s_{192+114}$ & 21.03 & $K_{35}$ & $s_{91} \Rightarrow s_{192+116}$ & 19.95 \\
\hline$\overline{K_{36}}$ & $s_{36} \Rightarrow s_{192+116}$ & 15.55 & $K_{37}$ & $s_{37} \Rightarrow s_{192+117}$ & 17.56 \\
\hline$\overline{K_{38}}$ & $s_{94} \Rightarrow s_{192+145}$ & 18.94 & $K_{39}$ & $s_{39} \Rightarrow s_{192+104}$ & 19.62 \\
\hline$K_{40}$ & $s_{152} \Rightarrow s_{192+60}$ & 16.43 & $K_{41}$ & $s_{153} \Rightarrow s_{192+116}$ & 17.90 \\
\hline$K_{42}$ & $s_{154} \Rightarrow s_{192+117}$ & 19.93 & $K_{43}$ & $s_{43} \Rightarrow s_{192+108}$ & 20.61 \\
\hline$K_{44}$ & $s_{156} \Rightarrow s_{192+145}$ & 16.90 & $K_{45}$ & $s_{157} \Rightarrow s_{192+146}$ & 18.96 \\
\hline$\overline{K_{46}}$ & $s_{46} \Rightarrow s_{192+35}$ & 20.45 & $K_{47}$ & $s_{47} \Rightarrow s_{192+6}$ & 16.68 \\
\hline$K_{48}$ & $s_{160} \Rightarrow s_{192+145}$ & 18.68 & $K_{49}$ & $s_{161} \Rightarrow s_{192+181}$ & 15.59 \\
\hline$K_{50}$ & $s_{162} \Rightarrow s_{192+182}$ & 17.59 & $K_{51}$ & $s_{51} \Rightarrow s_{192+116}$ & 15.62 \\
\hline$K_{52}$ & $s_{52} \Rightarrow s_{192+117}$ & 17.64 & $K_{53}$ & $s_{53} \Rightarrow s_{192+118}$ & 19.47 \\
\hline$K_{54}$ & $s_{54} \Rightarrow s_{192+119}$ & 20.05 & $K_{55}$ & $s_{55} \Rightarrow s_{192+120}$ & 20.61 \\
\hline$K_{56}$ & $s_{168} \Rightarrow s_{192+76}$ & 22.27 & $K_{57}$ & $s_{169} \Rightarrow s_{192+103}$ & 18.43 \\
\hline$K_{58}$ & $s_{170} \Rightarrow s_{192+104}$ & 18.17 & $K_{59}$ & $s_{171} \Rightarrow s_{192+105}$ & 18.93 \\
\hline$K_{60}$ & $s_{172} \Rightarrow s_{192+106}$ & 19.11 & $K_{61}$ & $s_{173} \Rightarrow s_{192+107}$ & 19.24 \\
\hline$\overline{K_{62}}$ & $s_{174} \Rightarrow s_{192+108}$ & 19.42 & $K_{63}$ & $s_{175} \Rightarrow s_{192+109}$ & 19.58 \\
\hline
\end{tabular}

IVs required to break DECIM with an 80-bit IV. 41 key bits can be recovered with less than $2^{21} \mathrm{IVs}$. Only the first 2 bytes of the keystream are required in the attack, and the amount of computation required in the attacks is negligible.

We explain Table 1 with $K_{0}$ as an example. $K_{0}$ is related to $s_{112}$ since $s_{112}=$ $K_{0} \oplus I V_{0} . s_{112}$ is mapped to $s_{192+60}$ with probability 0.0318 (this probability is obtained by tracing $s_{112}$ through the initialization process). Thus $K_{0}$ could be recovered by observing the first bits of the keystreams. About $2^{18.95} \mathrm{IVs}$ are required to achieve a success probability of 0.977 . 
Table 2. Number of IVs required to recover the key bits (80-bit IV)

\begin{tabular}{|c|c|c|c|c|c|}
\hline & Affected Bits & $\begin{array}{l}\text { Amount of } \\
\text { IVs }\left(\log _{2}\right)\end{array}$ & & Affected Bits & $\begin{array}{l}\text { Amount of } \\
\text { IVs }\left(\log _{2}\right)\end{array}$ \\
\hline$\overline{K_{0}}$ & $s_{32} \Rightarrow s_{192+183}$ & 20.70 & $\overline{K_{1}}$ & $s_{33} \Rightarrow s_{192+113}$ & 20.97 \\
\hline$K_{2}$ & $s_{34} \Rightarrow s_{192+114}$ & 21.03 & $\bar{K}$ & $s_{35} \Rightarrow s_{192+115}$ & 21.13 \\
\hline$K_{4}$ & $s_{36} \Rightarrow s_{192+116}$ & 15.55 & $K_{5}$ & $s_{37} \Rightarrow s_{192+117}$ & 17.56 \\
\hline$K_{6}$ & $s_{38} \Rightarrow s_{192+118}$ & 19.43 & $\overline{K_{7}}$ & $s_{39} \Rightarrow s_{192+104}$ & 19.62 \\
\hline$K_{8}$ & $s_{40} \Rightarrow s_{192+105}$ & 20.37 & $K_{9}$ & $s_{41} \Rightarrow s_{192+121}$ & 20.30 \\
\hline$K_{10}$ & $s_{42} \Rightarrow s_{192+107}$ & 20.48 & $K_{11}$ & $s_{43} \Rightarrow s_{192+108}$ & 20.61 \\
\hline$K_{12}$ & $s_{44} \Rightarrow s_{192+109}$ & 20.77 & $K_{13}$ & $s_{45} \Rightarrow s_{192+34}$ & 20.70 \\
\hline$K_{14}$ & $s_{46} \Rightarrow s_{192+35}$ & 20.45 & $K_{15}$ & $s_{47} \Rightarrow s_{192+6}$ & 16.68 \\
\hline$K_{16}$ & $s_{48} \Rightarrow s_{192+7}$ & 18.72 & $K_{17}$ & $s_{49} \Rightarrow s_{192+8}$ & 16.68 \\
\hline$K_{18}$ & $s_{50} \Rightarrow s_{192+9}$ & 18.66 & $K_{19}$ & $s_{51} \Rightarrow s_{192+116}$ & 15.62 \\
\hline$K_{20}$ & $s_{52} \Rightarrow s_{192+117}$ & 17.64 & $K_{21}$ & $s_{53} \Rightarrow s_{192+118}$ & 19.47 \\
\hline$\overline{K_{22}}$ & $s_{54} \Rightarrow s_{192+119}$ & 20.05 & $\overline{K_{23}}$ & $s_{55} \Rightarrow s_{192+120}$ & 20.61 \\
\hline$\overline{K_{24}}$ & $s_{56} \Rightarrow s_{192+121}$ & 20.63 & $K_{25}$ & $s_{57} \Rightarrow s_{192+122}$ & 20.83 \\
\hline$K_{26}$ & $s_{58} \Rightarrow s_{192+116}$ & 18.80 & $K_{27}$ & $s_{59} \Rightarrow s_{192+12}$ & 23.00 \\
\hline$K_{28}$ & $s_{60} \Rightarrow s_{192+13}$ & 23.41 & $K_{29}$ & $s_{61} \Rightarrow s_{192+14}$ & 23.66 \\
\hline$\overline{K_{30}}$ & $s_{62} \Rightarrow s_{192+15}$ & 23.78 & $K_{31}$ & $s_{63} \Rightarrow s_{192+16}$ & 24.09 \\
\hline$\overline{K_{32}}$ & $s_{64} \Rightarrow s_{192+17}$ & 24.00 & $\overline{K_{33}}$ & $s_{65} \Rightarrow s_{192+18}$ & 24.19 \\
\hline$K_{34}$ & $s_{66} \Rightarrow s_{192+19}$ & 24.22 & $K_{35}$ & $s_{67} \Rightarrow s_{192+5}$ & 23.44 \\
\hline$K_{36}$ & $s_{68} \Rightarrow s_{192+6}$ & 18.88 & $K_{37}$ & $s_{69} \Rightarrow s_{192+7}$ & 20.82 \\
\hline $\bar{K} 38$ & $s_{70} \Rightarrow s_{192+8}$ & 18.82 & $K_{39}$ & $s_{71} \Rightarrow s_{192+60}$ & 16.77 \\
\hline$K_{40}$ & $s_{72} \Rightarrow s_{192+61}$ & 18.75 & $K_{41}$ & $s_{73} \Rightarrow s_{192+62}$ & 20.59 \\
\hline$K_{42}$ & $s_{74} \Rightarrow s_{192+63}$ & 21.11 & $K_{43}$ & $s_{75} \Rightarrow s_{192+64}$ & 21.71 \\
\hline$\overline{K_{44}}$ & $s_{76} \Rightarrow s_{192+65}$ & 21.67 & $K_{45}$ & $s_{77} \Rightarrow s_{192+66}$ & 21.85 \\
\hline$K_{46}$ & $s_{78} \Rightarrow s_{192+67}$ & 21.81 & $K_{47}$ & $s_{79} \Rightarrow s_{192+145}$ & 18.82 \\
\hline$K_{48}$ & $s_{80} \Rightarrow s_{192+146}$ & 20.80 & $K_{49}$ & $s_{81} \Rightarrow s_{192+70}$ & 22.05 \\
\hline$K_{50}$ & $s_{82} \Rightarrow s_{192+71}$ & 22.18 & $K_{51}$ & $s_{83} \Rightarrow s_{192+72}$ & 22.40 \\
\hline$K_{52}$ & $s_{84} \Rightarrow s_{192+73}$ & 22.43 & $K_{53}$ & $s_{85} \Rightarrow s_{192+74}$ & 22.42 \\
\hline$K_{54}$ & $s_{86} \Rightarrow s_{192+75}$ & 22.43 & $K_{55}$ & $s_{87} \Rightarrow s_{192+76}$ & 22.55 \\
\hline$K_{56}$ & $s_{88} \Rightarrow s_{192+154}$ & 24.02 & $K_{57}$ & $s_{89} \Rightarrow s_{192+155}$ & 24.04 \\
\hline$K_{58}$ & $s_{90} \Rightarrow s_{192+156}$ & 24.15 & $K_{59}$ & $s_{91} \Rightarrow s_{192+116}$ & 19.95 \\
\hline$K_{60}$ & $s_{92} \Rightarrow s_{192+117}$ & 21.97 & $K_{61}$ & $s_{93} \Rightarrow s_{192+118}$ & 23.77 \\
\hline$\overline{K_{62}}$ & $s_{94} \Rightarrow s_{192+145}$ & 18.94 & $K_{63}$ & $s_{95} \Rightarrow s_{192+146}$ & 20.91 \\
\hline$K_{64}$ & $s_{96} \Rightarrow s_{192+147}$ & 22.79 & $K_{65}$ & $s_{97} \Rightarrow s_{192+148}$ & 23.33 \\
\hline$K_{66}$ & $s_{98} \Rightarrow s_{192+149}$ & 23.77 & $K_{67}$ & $s_{99} \Rightarrow s_{192+150}$ & 23.64 \\
\hline$K_{68}$ & $s_{100} \Rightarrow s_{192+63}$ & 22.65 & $K_{69}$ & $s_{101} \Rightarrow s_{192+4}$ & 23.12 \\
\hline$\overline{K_{70}}$ & $s_{102} \Rightarrow s_{192+65}$ & 23.66 & $K_{71}$ & $s_{103} \Rightarrow s_{192+178}$ & 23.80 \\
\hline$K_{72}$ & $s_{104} \Rightarrow s_{192+179}$ & 23.77 & $K_{73}$ & $s_{105} \Rightarrow s_{192+145}$ & 20.94 \\
\hline$K_{74}$ & $s_{106} \Rightarrow s_{192+181}$ & 18.24 & $K_{75}$ & $s_{107} \Rightarrow s_{192+182}$ & 19.97 \\
\hline$\overline{K_{76}}$ & $s_{108} \Rightarrow s_{192+183}$ & 21.81 & $K_{77}$ & $s_{109} \Rightarrow s_{192+6}$ & 20.86 \\
\hline$K_{78}$ & $s_{110} \Rightarrow s_{192+7}$ & 22.83 & $K_{79}$ & $s_{111} \Rightarrow s_{192+8}$ & 20.94 \\
\hline
\end{tabular}

